\title{
Bounds on entanglement in qudit subsystems
}

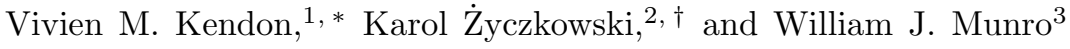 \\ ${ }^{1}$ Optics Section, Blackett Laboratory, Imperial College, London, SWr 2BW, United Kingdom. \\ ${ }^{2}$ Center for Theoretical Physics, Polish Academy of Sciences, Al. Lotników 32/44, 02-668 Warszawa, Poland \\ ${ }^{3}$ Hewlett-Packard Laboratories, Filton Road, Stoke Gifford, Bristol, BS34 8QZ, United Kingdom.
}

(Dated: March 18, 2002; revised May 30, 2002; further revised September 17, 2002)

\begin{abstract}
The entanglement in a pure state of $N$ qudits ( $d$-dimensional distinguishable quantum particles) can be characterized by specifying how entangled its subsystems are. A generally mixed subsystem of $m$ qudits is obtained by tracing over the other $N-m$ qudits. We examine the entanglement in the space of mixed states of $m$ qudits. We show that for a typical pure state of $N$ qudits, its subsystems smaller than $N / 3$ qudits will have a positive partial transpose and hence are separable or bound entangled. Additionally, our numerical results show that the probability of finding entangled subsystems smaller than $N / 3$ falls exponentially in the dimension of the Hilbert space. The bulk of pure state Hilbert space thus consists of highly entangled states with multipartite entanglement encompassing at least a third of the qudits in the pure state.
\end{abstract}

PACS numbers: 03.67.-a, 03.65.Ud, 03.67.Lx

\section{INTRODUCTION}

Quantum information is a rapidly developing field exploiting the peculiar entanglement properties of quantum states. Its applications can be broadly divided into two general types: quantum computation [1, 2], and quantum communication [3]. Quantum communication (including quantum cryptography and quantum teleportation) can be framed in terms of repeated use of pairs of entangled qubits - the entanglement properties of two qubits have been well characterized, and a number of analytical

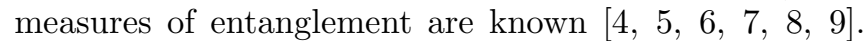
However, for multi-qubit systems, which are essential for quantum computation, few entanglement measures can be calculated even for pure states. Despite these difficulties, arrays of qubits have been the focus of recent attention 110, 11, 12, 13, 14, 15, though often only pairwise entanglement has been considered in such systems. The more general question of entanglement in multi-qudit systems ( $d$-dimensional quantum particles) is also important since most real systems (e. g., atoms) have more than two states.

In this paper we tackle the question of entanglement in larger qudit systems by deriving a bound on the size of typical entangled mixed states that are subsystems of larger pure states. Previous numerical work by some of us [16] suggested that qubit subsystems somewhat less than half the size of the pure state, typically have no usable entanglement. In other words, since pure states are typically highly entangled, the entanglement is distributed in multi-partite entanglement involving around half or more of the qubits. This work was limited by computational resources to small numbers of qubits (less than 14). Here we provide analytical bounds for any number of qubits,

\footnotetext{
*Electronic address: Viv.Kendon@ic.ac.uk

${ }^{\dagger}$ Electronic address: karol@cft.edu.p
}

and also extend the analysis to any dimension of qudits.

We emphasize that we are interested in average properties of all possible pure states. It is easy to construct states with entanglement properties that lie outside our bounds. For example, the so-called $W$ states (symmetric superposition of one qubit in state $|1\rangle$ with the rest in state $|0\rangle$ ) have only pairwise entanglement 15 for any value of $N$. However, such states are of small measure and do not contribute significantly to the average properties of pure states sampled from the whole of Hilbert space.

To further motivate our investigations, we note a connection to the the question of the origin of the speedup in quantum computing. Recently Jozsa and Linden in 117] argue that for an exponential speedup over classical computation, the state of a quantum computer using pure states will have multi-partite entanglement encompassing an arbitrarily large fraction of the total number of qubits in the computation. However, they also point out that the entanglement cannot be inferred to be the cause of the speedup, rather, it is one of a number of properties of the typical states in the Hilbert space of the quantum computer that are necessary for such a speed up. Our work confirms this property of typical states in Hilbert space, and also provides an analytical lower bound on the number of parties in the multi-partite entanglement for a given size of Hilbert space.

The paper is organized as follows. First, in Sec. II, we introduce clear definitions of the system we are considering and the entanglement measures we will use to analyze it. Next in Sec. [III, we derive the analytic bounds, followed by comparison with numerical results in Sec. IV. We finish with conclusions in Sec. V. 


\section{DEFINITIONS AND ENTANGLEMENT MEASURES}

In a finite dimensional Hilbert space $\mathcal{H}$ there exists a natural, unitarily invariant, Fubini-Study measure $\mu_{F S}$, induced by the Haar measure on the unitary group. A system of $N$ qudits has a Hilbert space of size $d^{N}$. We will investigate entanglement in such systems by sampling randomly from the set of all pure $N$-qudit states according to the natural measure $\mu_{F S}$ and calculating their average, which is to say, typical properties. This will give us useful information about the majority of states in Hilbert space. Having chosen a random pure state of $N$ qudits, we can divide it into two subsystems by partial tracing. A subsystem of size $m$ is obtained by partial tracing over the other $N-m$ qudits. This partitioning could represent the system of interest plus the environment, for example, or two different parts of one system. From the mathematical point of view one obtains in this way a certain probability measure $\mu_{N, m}$ in the space of mixed quantum states acting in a $d^{m}$ dimensional Hilbert space induced by the natural measure $\mu_{F S}$ on the space of pure states in $\mathcal{H}_{d^{N}}$ [18]. This is a useful way to sample randomly from mixed states, since unlike $\mu_{F S}$ for pure states, there is no unique natural measure for the space of mixed states.

\section{A. Entropy of subsystems}

Consider now a pure state quantum system described by a density operator $\rho=|\phi\rangle\langle\phi|$ acting in a composite Hilbert space $\mathcal{H}_{M K}=\mathcal{H}_{M} \otimes \mathcal{H}_{K}$ of dimension $M K$. The reduced density matrices defined by the partial trace, $\rho_{M}:=\operatorname{tr}_{K} \rho$ and $\rho_{K}:=\operatorname{tr}_{M} \rho$ characterize both subsystems. It is well-known that the von Neuman entropies of both subsystems are equal, $S_{M}=S\left(\rho_{M}\right)=$ $-\operatorname{tr} \rho_{M} \ln \rho_{M}=S\left(\rho_{K}\right)=S_{K}$. The value of $S_{M}$ shows how entangled the two subsystems are with each other. If $S_{M}=0$, there is no entanglement and the composite state may be factorized, $\rho=\rho_{M} \otimes \rho_{K}$, but this is not a typical case. The mean entropy of a subsystem averaged over the natural measure $\mu_{F S}$ is given by

$$
\left\langle S_{M}\right\rangle=\sum_{j=K+1}^{M K} \frac{1}{j}-\frac{M-1}{2 K} \simeq \ln M-\frac{M}{2 K},
$$

where $M \leq K$ and the approximation holds for $0 \ll$ $M<K$. This result was first conjectured by Page 19$]$ and later proved in 20, 21.

In our case the subsystems consist of $m$ and $N-m$ qudits, so substituting $M=d^{m}$ and $K=d^{N-m}$ into (11) we obtain the mean entropy $\langle S\rangle_{N, m}$ of the subsystem analyzed, where we are now using the subscripts to remind us of the number of qudits in the pure state $(N)$ as well as the number in the subsystem $(m)$. Equation (1) tells us that on average, the smaller subsystem has nearly maximal entropy, showing that the two subsystems are highly entangled with each other, a typical pure state is highly entangled, see also [22]. The particular value of $S_{N, m}$ provides a good measure of entanglement between the two subsystems, but says little about entanglement between the qudits within a single subsystem. Asymptotically, for $(N \rightarrow \infty), m \leq N / 2$ will tend to be maximally mixed and therefore separable, but to obtain useful results for finite $N$, we need further information on the size of the region around the maximally mixed state that contains separable states.

\section{B. Purity and mixedness}

Next we will characterize how mixed a typical $m$-qudit state $\rho_{m}$ might be. A convenient measure is the purity [23] defined by

$$
r\left(\rho_{m}\right):=\operatorname{tr}\left(\rho_{m}^{2}\right) .
$$

Pure states are defined to have $r\left(\rho_{m}\right)=1$ while mixed states have $1 / d^{m} \leq r\left(\rho_{m}\right)<1$. We will also refer to $R:=1 / \operatorname{tr}\left(\rho_{m}^{2}\right) \equiv 1 / R$ as the inverse participation ratio (IPR) of the mixed state $\rho_{m}$. The IPR thus ranges from 1 (pure) to $d^{m}$ (maximally mixed), and larger IPR means the states are more mixed, while larger purity means the states are more nearly pure.

Consider random states drawn according to the natural measure $\mu_{F S}$ on the space of pure states in a $M K$ dimensional Hilbert space. As before, we apply a partial trace to obtain the reduced density matrices $\rho_{M}$ and $\rho_{K}$. In refs. [18, 24, 25] it was shown that the average purity of $\rho_{M}$ and $\rho_{K}$ is equal to

$$
\langle r\rangle=\frac{M+K}{M K+1}
$$

This result does not depend on the particular way in which the $M K$-dimensional Hilbert space is decomposed, but only on the initial and the final dimensionality of the spaces. Thus it also holds in the problem we are analyzing here of pure states of $N$-qudits reduced to $m^{-}$ qudits by partial tracing. Substituting $M=d^{m}$ and $K=d^{N-m}$ the average purity of the system reads

$$
\langle r\rangle_{N, m}=\frac{d^{m}+d^{N-m}}{d^{N}+1}
$$

In other words, the averaging has been performed with respect to the induced measure $\mu_{N, m}$.

Numerical investigation of the induced measures shows 18] that for $m \lesssim N / 2$ the probability distributions $P(r)$ are concentrated close to the mean value $\langle r\rangle$. This is illustrated in fig. 1, where $P(r)$ is plotted for $d=m=2$ and $N=3$ to 6 in the main plot, and for $d=2, m=3$, $N=5$ to 10 in the inset. The scale of the purity axis on the inset has been adjusted (the scale is in fact linear in $R=1 / r$ ) to show the data near the minimum purity more clearly. 


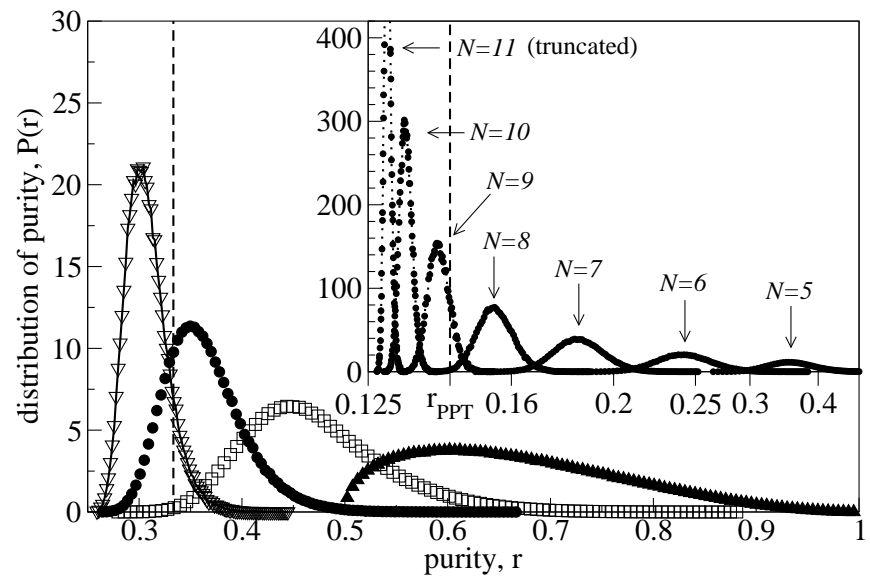

FIG. 1: The numerically obtained distribution of the purity $r$ for (main graph) mixed states of $m=2$ qubits obtained by partial tracing of random pure states of (right to left) $N=3$, 4, 5 and 6 qubits, and (inset) mixed states of $m=3$ qubits for $N=5$ to 10 . The vertical dashed lines indicate the values of $r_{\mathrm{PPT}}$ from eq. (5).

Fixing the number $m$ of the qudits in the final system and varying the initial number $N$ of qudits involved in the pure state we get a sequence of probability measures which sweep the space of the mixed states close to the manifold of a constant purity. Hence it is justified to use the mean value $\langle r\rangle$ as a good estimate of the actual value $r$ for a typical subsystem.

\section{Peres criterion, positive partial transpose}

We will now describe a simple test for entanglement in multiparticle mixed states due to Peres [26]. Consider a multiparticle mixed state $\rho$ acting in a $d^{m}$ dimensional Hilbert space, which describes the system consisting of $m$ qudits. An $m$-particle system can be split into two parts containing $j$ and $m-j$ particles respectively. The partial transpose is applied to the $j$ particle part of the system producing $\rho^{T_{j}}:=\left(\mathbf{1}_{L} \otimes T_{j}\right) \rho$. Here $T_{j}$ denotes the transpose operation in the $d^{j}$ dimensional subspace, while the dimension of the subspace that has not been transposed is $L=d^{m-j}$. We say that the $m$-qudit state has the PPT property, if $\rho^{T_{j}}$ is positive for all possible values of $j$ (it is sufficient to take $1 \leq j \leq m / 2$ ) and choices of $j$ particles, i. e., with respect to all of its possible splitting into two subsystems. Conversely, a state which fails this test is described as being NPT (negative partial transpose). NPT states have some useful entanglement that could be distilled with some probability into one or more maximally entangled states of two qubits. This test does not distinguish bound entangled states 27, 28] from separable states, but since bound entanglement is relatively rare [29], and for most purposes it is the free entanglement that is useful, this still provides a useful characterization of entanglement.

For bipartite systems with Hilbert space dimension $d^{2}$, it has been shown [5] that all the states sufficiently close to the maximally mixed state, with $R \geq d^{2}-1$ have a positive partial transpose (PPT). This condition defines the maximal ball inscribed in the convex body of mixed states. Very recently it was shown that all mixed states of a bipartite system belonging to the maximal ball posses not only the PPT property but are also separable [30. The former result may be generalized for a multipartite case: any state $\rho_{m}$ (within the $d^{m}$ dimensional Hilbert space) for which

$$
R \geq R_{\mathrm{PPT}}=d^{m}-1 \quad \text { so that } \quad r \leq r_{\mathrm{PPT}}=\frac{1}{d^{m}-1}
$$

has the PPT property with respect to any possible transpositions of the subsystems. We give the proof of the generalization in Appendix A.

\section{BOUNDS ON ENTANGLEMENT IN SUBSYSTEMS}

We are now ready to provide an analytical relationship between the size of a subsystem and its typical entanglement properties.

\section{A. Bound on PPT region}

Using the fact that the distributions of the purity $P(r)$ are narrow for the regions of interest $(m \lesssim N / 2)$, and thus $\langle r\rangle_{N, m}$ is a good estimate of the actual value of $r$ in a typical subsystem, we apply this to the critical value $r_{\mathrm{PPT}}$ obtained in the previous section. If the mean value $\langle r\rangle$ averaged over a certain induced measure is smaller that the critical value $r_{\mathrm{PPT}}$, (or $\langle R\rangle>R_{\mathrm{PPT}}$ ), the majority of random states distributed with respect to this measure are localized inside the maximal ball and are PPT. We therefore combine eqs. (5) and (4) to calculate a relationship between $N$ and $m$ such that we expect almost all subsystems of $m$ qudits will have a positive partial transpose. We find $\langle r\rangle_{N, m} \leq r_{\mathrm{PPT}}$ when

$$
d^{N} \geq d^{3 m}\left(1-d^{-m}-d^{-2 m}\right) .
$$

Taking the logarithm (base $d$ ) gives us an estimate of how many qudits the initial pure state should contain, such that after the reduction to $m$-qudits the obtained mixed state is PPT on average,

$$
N_{\mathrm{PPT}} \geq 3 m+\log _{d}\left(1-d^{-m}-d^{-2 m}\right) .
$$

The $\log _{d}$ term is always negative, becoming rapidly smaller for either $m>2$ or $d>2$. Hence for systems initially consisting of

$$
N \geq 3 m
$$

qudits we expect the subsystems of size $m$ to be PPT mixed states with a considerable probability. For the 
simplest case of $m=d=2$, the smallest integer number larger than the right hand side of (7) gives $N_{\mathrm{PPT}}=$ $\operatorname{int}\left[6-\log _{2}(16 / 11)\right]+1=6$. For a fixed number $N$ this establishes a bound on $m$ below which subsystems of this size have on average no useful entanglement in an $N$ qudit pure state. This is the main result in this paper.

It is also possible to calculate the maximum value of the von Neuman entropy for states on the surface of the maximal ball, see Appendix B. This value $S_{c}$, given in eq. (B1), can then be combined with eq. (11) for the average entropy of a subsystem, to obtain a similar relationship between $N$ and $m$. The result is (as it should be) the same as eq. (8), but with the restriction $m \gg 0$ from the approximate form of eq. (11). Our derivation as presented using eqs. (5) and (4) is applicable to all $0<m<N$.

\section{B. Estimation of the transition region}

The above result is a bound on the average properties of typical states that identifies the range of parameter values $(N \geq 3 m)$ where there is a high probability that a subsystem of size $m$ is PPT. We would also like to characterize the range of $N, m$ for which with high probability the subsystems of size $m$ are entangled. This region is not simply the inverse of the PPT region because while the criterion we are using in eq. (5) is sufficient for ensuring the subsystem is PPT, it is not a necessary condition. There are many states which are PPT that have $r>r_{\mathrm{PPT}}$ right up to separable pure states with $r=1$.

Since we do not, in general, know the size of the region of PPT states outside the maximal ball defined by eq. (5), our approach is to identify a region of the body of mixed states of a considerable size, which contains entangled states only. There is a one parameter family of mixed states (known as the generalized Werner states) containing $m$ qudits defined as a mixture of the maximally entangled pure state $|\Psi\rangle$ and the maximally mixed state $I_{d^{m}}$,

$$
\rho_{\varepsilon}=\frac{(1-\varepsilon)}{d^{m}} I_{d^{m}}+\varepsilon|\Psi\rangle\langle\Psi|
$$

where

$$
|\Psi\rangle=\frac{1}{\sqrt{d}} \sum_{i=1}^{d}|i\rangle_{1} \otimes \ldots \otimes|i\rangle_{m}
$$

and $\varepsilon$ is a real parameter between 0 and 1 specifying the proportions of the mixture. These states have been shown to be entangled for 31, 32, 33.

$$
\varepsilon>\varepsilon_{\text {ent }}=\frac{1}{d^{m-1}+1} .
$$

and strictly separable for $\varepsilon \leq \varepsilon_{\text {ent }}$ (there are no bound entangled states in this family). This boundary at $\varepsilon_{\text {ent }}$ defines a set of entangled states of positive measure [39] as can be seen as follows. The states $\rho_{\varepsilon}$ lie on a line that is on an axis of rotational symmetry in the set of mixed states. This line intersects the set of separable states at $\varepsilon=\varepsilon_{\text {ent }}$. The set of separable states is convex, so beyond the hyperplane normal to the $\rho_{\varepsilon}$ line there exists a set of entangled states of a positive measure (see also [34]).

It is straight forward to show that the purity for $\rho_{\varepsilon}$ at this boundary point $\left(\varepsilon=\varepsilon_{\text {ent }}\right)$ is given by

$$
r_{\text {ent }}=\frac{d^{m}+d^{2}+2 d}{\left(d^{m}+d\right)^{2}} .
$$

Thus there exists a greater-than-zero probability to encounter entangled states with $r>r_{\text {ent }}$. However, we don't know that entangled states actually dominate over PPT states for this value of $r$. Since Werner states are among the most entangled states [35, 36, 37 for a given purity $r$, we might actually guess that this estimate will turn out to be too tight. Nonetheless, it provides a useful independent check on our previously derived bound, so we present it anyway.

Equating $\langle r\rangle_{N, m}$ from eq. (4) with $r_{e n t}$ from (12) allows us to establish an estimate for the entangled side of the transition between entangled and PPT states. It is easily shown that $\langle r\rangle_{N, m} \geq r_{\text {ent }}$ when

$$
N_{\text {ent }} \leq 3 m-2+\log _{d}\left[1+(2 d+1) d^{-m}+(d+2) d^{1-2 m}\right] .
$$

For all $d$ and $m$, the $\log _{d}$ term is positive and tending to zero for increasing $d$ and $m$. Hence for all finite $N$ with

$$
N \leq 3 m-2
$$

we expect the probability of finding the subsystem of size $m$ entangled (not PPT), to be non-zero. Looking at the smallest case [40], $d=m=2$, we have $N_{\text {ent }}=$ $\operatorname{int}\left[4+\log _{2}(11 / 4)\right]=5$. Let us emphasize again, that this is an estimation only; for this (or lower) values of $N$ the probability of finding entangled subsystems of size $m$ is non-zero, but it does not rule out the existence of substantial numbers of PPT subsystems of size $m$, so the transition might occur effectively for even smaller values of $N$.

Now let us fix the initial size of the pure states of $N$ qudits while we decrease the size of the final system of $m$ qudits. For $m=N$ the probability of finding a separable pure state is equal to zero. As $m$ is reduced, the states obtained by partial tracing over $N-m$ qudits become increasingly mixed, and the probability of finding PPT states increases. Putting both results together we may characterize quantitatively a transition region

$$
3 m-2 \lesssim N \leq 3 m,
$$

which does not depend on the qudit size $d$, in which we estimate that PPT subsystems come to dominate over entangled subsystems. (Note that the inequalities have reversed because we are identifying the region where the ratio of PPT to NPT (negative partial transpose) subsystems of size $m$ is of order unity, rather than the regions 


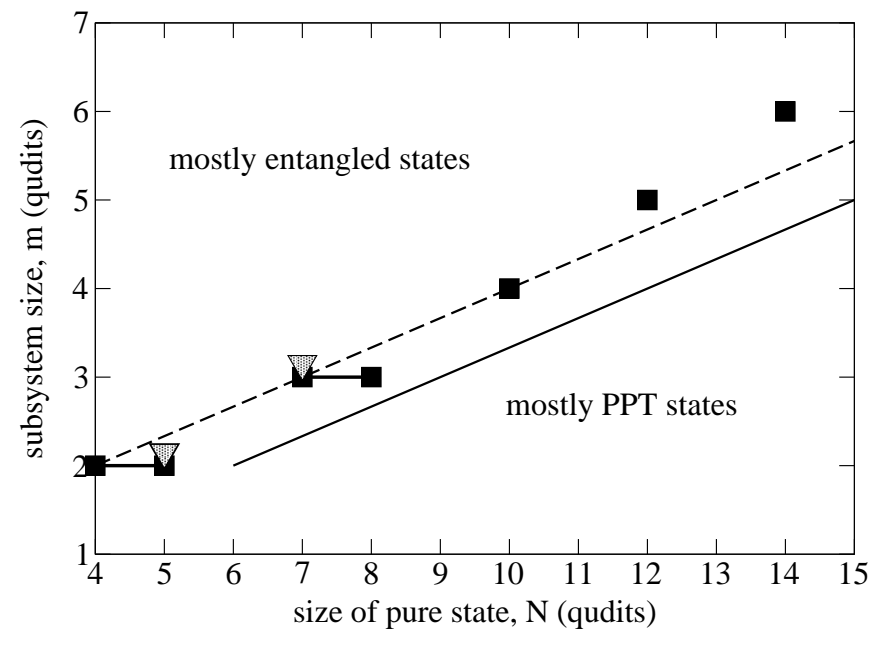

FIG. 2: Numerical results showing the transition from entangled to PPT subsystems. For each $m$, points are shown for which $P_{\text {PPT }} \in(1 \%, 99 \%)$ for qubits $(\mathbf{\square})$ and qutrits (grey $\nabla$ ). The solid/dashed lines represent the range in eq. (15).

where one case dominates over the other.) These are surprisingly tight estimates of the boundary between entangled and PPT states in terms of the number of qudits in the state. However, it must be remembered that the number of qudits is a logarithmic measure of the size of the Hilbert space. The addition of one qudit enlarges the Hilbert space by a factor of $d$.

\section{NUMERICAL COMPARISONS}

In [16], for the qubit case $d=2$, it was shown numerically that entanglement in a mixed $m$-qubit subsystem of a typical $N$-qubit pure state falls off sharply towards zero with increasing $N$ for values of $m \leq 5$ and $N \leq 13$. These numerical results suggest the transition from NPT states dominating to PPT dominanting is complete by $N \simeq 2 m+3$, which falls outside the range of eq. (15) for $m>4$. This is at the edge of the results presented in [16], so we ran further numerical studies to confirm this divergence between numerical and analytical results and also, since our analytical results apply for any dimension of the constituent quantum particles $d$, to test qutrits and confirm they follow the same pattern.

Using straightforward C programs optimized for efficiency to generate the large data sets required for useful statistics, we generated random pure states in the $d^{N}$ dimensional Hilbert space, traced over $N-m$ qudits, and analyzed whether the remaining mixed state, with a $d^{m}$-dimensional Hilbert space, had a positive or negative partial transpose (PPT or NPT). In fig. 2 we represent the transition region in the $N-m$ plane for qubits up to $m=6, N=15$ and qutrits up to $m=3, N=8$, combining data from [16] with our new data for $N=14$ to 15 (qubits) covering the transition for $m=6$, and qutrits

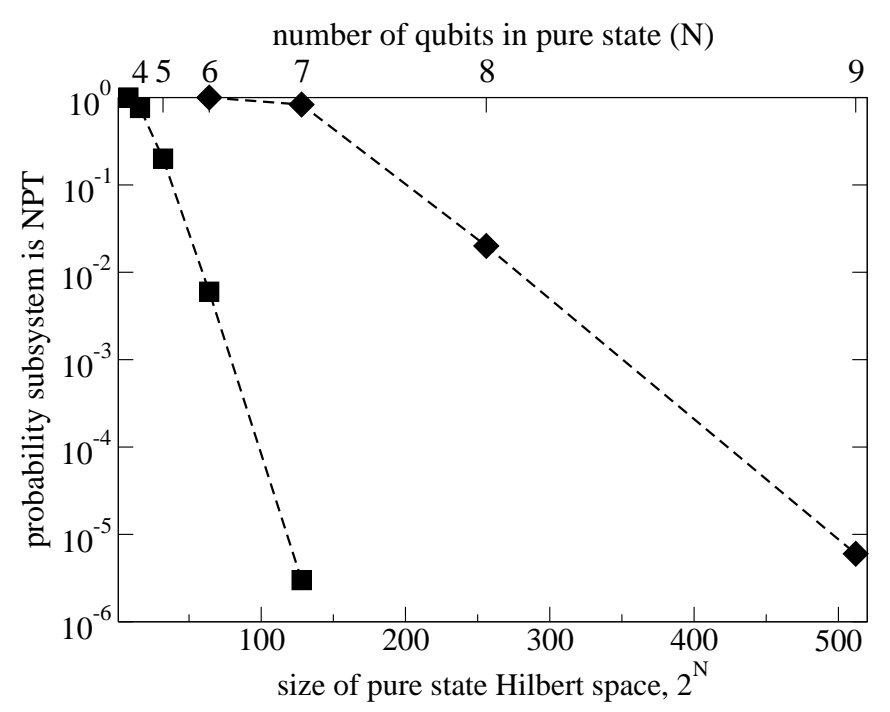

FIG. 3: Probability of finding that a subsystem of $m$ qudits is entangled in random pure states sampled uniformly over the Haar measure for qubits for $m=2(\boldsymbol{\square})$ and $m=3$ (filled $\diamond$ ).

for $N=4$ to 8 . Our new results confirm the trend. For $m>4$, the transition from NPT to PPT for a given subsystem size $m$ is completed for $N<3 m-2$. This is due to our estimates not taking into account the full size of the set of PPT states, being based only on the maximal ball inscribed in this set.

There is one more feature of this transition that requires comment. As noted in 16, the transition is very sharp, the proportion of PPT subsystems of a given size $m$ becomes essentially zero to numerical accuracy with the addition of just a few qudits to the pure state. It is not obvious a priori that the transition should be sharp. One might reasonably expect it to be smoother, with a typical pure state of $N$ qudits containing finite amounts of $m$-partite entanglement for all $2 \leq m \leq N$. In order to obtain such a sharp transition, the proportion of NPT subsystems of given size $m$ must fall exponentially in the size of the Hilbert space $d^{N}$ of the pure state. This is hard to check numerically, but the data we have do support this, see fig. 3. This sharp transition and exponential fall off allows us to assert that this bound on the multi-partite nature of entanglement in typical states of Hilbert space corresponds to the distinction made in [17] between sets of states that can be simulated efficiently classically, and sets of states that cannot. It also justifies our statement in the introduction that exceptions to our bound (8) do not contribute significantly to the average properties of typical pure states.

\section{DISCUSSION AND CONCLUSIONS}

We have obtained analytically a lower bound on the size of subsystem of a typical pure state that can be 
expected to contain any entanglement within the subsystem. For a typical pure state of $N$ qudits, its subsystems of size $m<N / 3$ can be expected to be PPT, i.e. to have no usable entanglement within them. We then checked numerically and confirmed that the transition from NPT to PPT subsystems actually occurs at significantly larger $m$ nearer to $N / 2$, which is the asymptotic limit as $N \rightarrow \infty$. Our results emphasize the importance of multipartite entanglement in quantum information processing. The types of states necessary for exploring the main bulk of Hilbert space with quantum computers are highly entangled, but the entanglement will not be evident if only a small subset of the qudits are examined. For a quantum computer operating in a pure state, at least a third of the qubits would need to be processed to detect the entanglement present.

\section{Acknowledgments}

It is a pleasure to thank Christof Zalka for useful discussions. We also thank the referee for helpful comments. This work was funded in part by the European project EQUIP(IST-1999-11053) and QUIPROCONE (IST-1999-29064). VK is funded by the UK Engineering and Physical Sciences Research Council grant number GR/N2507701. KŻ acknowledges the Polish KBN grant no 2P03B-072-19 and is grateful to the Blackett Laboratory for the hospitality during his stay in London.

\section{APPENDIX A: PPT PROPERTY FOR MIXED STATES OF MULTI-PARTITE SYSTEMS}

In this appendix we prove the following

Proposition. Any mixed state $\rho$ of $m$ qudits which satisfies the condition

$$
R(\rho)=\frac{1}{\operatorname{tr} \rho^{2}} \geq d^{m}-1
$$

has the PPT property, $i$. e., all its partially transposed matrices $\rho_{m}^{T_{j}}$ are positive.

We start the proof by invoking an algebraic

Lemma. Let $A$ be a $n \times n$ non-zero Hermitian matrix and consider the real number $\alpha:=\operatorname{tr} A /\left(\operatorname{tr} A^{2}\right)^{1 / 2}$. If $\alpha \geq \sqrt{n-1}$ then $A \geq 0$

the proof of which is given by Mehta, 38], inequality 9.21 , p. 217. Let us apply it for the analyzed density matrix partially transposed in an arbitrary way, $A=$ $\rho^{T_{j}}$, which remains a Hermitian matrix of size $n=d^{m}$. Any operation of partial transpose $T_{j}$ does not change the traces, so $\operatorname{tr} \rho^{T_{j}}=\operatorname{tr} \rho=1$ and $\operatorname{tr}\left(\rho^{T_{j}}\right)^{2}=\operatorname{tr} \rho^{2}=$ $1 / R$. Therefore, the coefficient $\alpha^{2}=1 / \operatorname{tr} \rho^{2}$ is just equal to the inverse participation ratio $R$. If a mixed state $\rho$ satisfies the condition (A1), the assumption $\alpha \geq \sqrt{n-1}$ is fulfilled. Hence matrices $\rho^{T_{j}}$ are positive for all possible operations $T_{j}$ of partial transpose, so the state $\rho$ is PPT. $\square$.

It is illuminating to discuss a simple geometric interpretation of the condition (A1), which defines a certain subset of the convex body of mixed states $\mathcal{M}$ acting in $n=d^{m}$ dimensional Hilbert space. The center of the $n^{2}-1$ dimensional set $\mathcal{M}$ is given by the maximally mixed state $\rho_{*}=\mathbb{I} / n$. Introducing the Hilbert-Schmidt metric

$$
D_{\mathrm{HS}}\left(\rho_{1}, \rho_{2}\right)=\left(\operatorname{tr}\left(\rho_{1}-\rho_{2}\right)^{2}\right)^{1 / 2}
$$

it is not difficult to compute the distance of any state $\rho$ with eigenvalues $\left\{x_{i}\right\}$ to the center of $\mathcal{M}$,

$$
D_{H S}^{2}\left(\rho, \rho_{*}\right)=\sum_{i=1}^{n}\left(x_{i}-\frac{1}{n}\right)^{2}=\frac{1}{R}-\frac{1}{n} .
$$

Hence the condition $R=$ const determines a set of points equidistant from $\rho_{*}$.

The boundary of $\mathcal{M}$ is defined by the condition $\operatorname{det} \rho=$ 0 , so any state belonging to it has at least one eigenvalue equal to zero. The state $\rho_{a}$ with the spectrum $\left\{\frac{1}{n-1}, \frac{1}{n-1}, \ldots, \frac{1}{n-1}, 0\right\}$ is closest to $\rho_{*}$ among the states belonging to the boundary of $\mathcal{M}$. Its inverse participation ratio, $R\left(\rho_{a}\right)=n-1$, coincides with the critical value from the constraint (A1). Thus all states satisfying it form the maximal ball inscribed into the convex body of mixed states $\mathcal{M}$ and centered at $\rho_{*}$.

\section{APPENDIX B: VON NEUMANN ENTROPY ON THE BOUNDARY OF THE MAXIMAL BALL}

In this appendix we outline the proof of the following

Proposition. If the von Neuman entropy of a state $\rho$ is larger than $S_{c}$, then $\rho$ belongs to the maximal ball and its inverse participation ratio $R$ is larger than $n-1$ where $n$ is the dimension of the Hilbert space of $\rho$ and $S_{c}$ is given by

$$
S_{c}=\ln n-\frac{2}{n} \ln 2+\frac{(n-2)}{n} \ln \left[\frac{n-1}{n-2}\right] .
$$

In the asymptotic limit $n \rightarrow \infty, S_{C} \rightarrow \ln n$, i.e. maximal.

State $\rho_{a}$, with the spectrum

$$
\lambda_{a}=\left\{\frac{1}{n-1}, \ldots \frac{1}{n-1}, 0\right\},
$$

belongs to the boundary of the set of mixed states and to the maximal ball inscribed in it since

$$
R=\frac{1}{\operatorname{tr}\left(\rho^{2}\right)}=n-1,
$$

see Appendix A. Its entropy $S\left(\rho_{a}\right)=\ln (n-1)$ is the smallest among all states located at the surface of the hyperspehere $R=n-1$. To show this it suffices to perturb the spectrum keeping $R$ constant and compute the 
entropy variation. Due to the symmetry of the problem, the state $\rho_{b}$ which belongs to this hypersphere and has the largest entropy, is located opposite to $\rho_{a}$ with respect to the maximally mixed state $\rho_{*}=\mathbb{I} / n$ and we have

$$
\left(\rho_{b}+\rho_{a}\right) / 2=\rho_{*} .
$$

This gives the spectrum of $\rho_{b}$

$$
\lambda_{b}=\left\{\frac{n-2}{n(n-1)}, \ldots, \frac{n-2}{n(n-1)}, \frac{2}{n}\right\},
$$

which evidently belongs to the maximal ball $R=n-1$.

Computing its entropy $S\left(\rho_{b}\right)$ one obtains the critical value $S_{c}$ given by $(\mathrm{B} 1)$. $\square$.
[1] D. P. DiVincenzo, Science 270, 255 (1995).

[2] V. Vedral and M. B. Plenio, Prog. Quant. Electron. 22, 1 (1998).

[3] B. Schumacher, Phys. Rev. A 54, 2614 (1996).

[4] W. K. Wootters, Phys. Rev. Lett. 80, 2245 (1998), quant$\mathrm{ph} / 9709029$.

[5] K. Życzkowski, P. Horodecki, A. Sanpera, and M. Lewenstein, Phys. Rev. A 58, 883 (1998), quant-ph/9804024.

[6] M. Horodecki, Quant. Inf. Comp. 1, 3 (2001).

[7] G. Vidal and R. F. Werner, Phys. Rev. A 65, 032314 (2002), quant-ph/0102117.

[8] C. H. Bennett, D. P. DiVincenzo, J. A. Smolin, and W. K. Wootters, Phys. Rev. A 54, 3824 (1996).

[9] V. Vedral, M. B. Plenio, M. A. Rippin, and P. L. Knight, Phys. Rev. Lett. 78, 2275 (1997).

[10] P. Stelmachovic and V. Bužek, Entanglement in the Ising model (2001).

[11] R. Raussendorf and H. J. Briegel, Phys. Rev. Lett. 86, 5188 (2001).

[12] D. Gunlycke, V. M. Kendon, V. Vedral, and S. Bose, Phys. Rev. A 64, 042302 (2001), quant-ph/0102137.

[13] W. K. Wootters, Entangled chains (2000), quant$\mathrm{ph} / 0001114$.

[14] K. M. O'Connor and W. K. Wootters, Phys. Rev. A 63, 052302 (2001), quant-ph/0009041.

[15] M. Koashi, V. Bužek, and N. Imoto, Phys. Rev. A 62, 050302(R) (2000), quant-ph/0007086.

[16] V. M. Kendon, K. Nemoto, and W. J. Munro, J. Mod. Optics 49(10), 1709 (2002), quant-ph/0106023.

[17] R. Jozsa and N. Linden, On the role of entanglement in quantum computational speed-up (2002), quant$\mathrm{ph} / 0201143$.

[18] K. Życzkowski and H.-J. Sommers, J. Phys. A: Math. Gen 34, 7111 (2001), quant-ph/0012101.

[19] D. N. Page, Phys. Rev. Lett 71, 1291 (1993), grqc/9305007.

[20] S. Sen, Phys. Rev. Lett. 77, 1 (1996), hep-th/9601132.

[21] S. K. Foong and S. Kanno, Phys. Rev. Lett. 72, 1148 (1994).

[22] M. A. Nielsen and J. Kempe, Phys. Rev. Lett. 86(22), 5184 (2001), quant-ph/0011117.

[23] D. Weaire and A. R. Williams, J. Phys. C: Solid State 10, 1239 (1977).

[24] P. Zanardi, C. Zalka, and L. Faoro, Phys. Rev. A 62, 030301(R) (2000), quant-ph/0005031.

[25] E. Lubkin, J. Math. Phys. 19, 1028 (1978).

[26] A. Peres, Phys. Rev. Lett. 77, 1413 (1996), quant- $\mathrm{ph} / 9604005$.

[27] M. Horodecki, P. Horodecki, and R. Horodecki, Phys. Rev. Lett. 80, 5239 (1998), quant-ph/9801069.

[28] P. Horodecki, Phys. Lett. A 232, 333 (1997), quant$\mathrm{ph} / 9703004$.

[29] K. Życzkowski, Phys. Rev. A 60, 3496 (1999), quant$\mathrm{ph} / 9902050$.

[30] L. Gurvits and H. Barnum, Size of the separable neighbourhood of the maximally mixed bipartite state (2002), quant-ph/0204159.

[31] P. Rungta, W. J. Munro, K. Nemoto, P. Deuar, G. J. Milburn, and C. M. Caves, Directions in Quantum Optics: A Collection of Papers Dedicated to the Memory of Dan Walls edited by H. J. Carmichael, R. J. Glauber and M. O. Scully, Springer-Verlag, Berlin pp. 149-164 (2001), quant-ph/0001075.

[32] P. Deuar, W. J. Munro, and K. Nemoto, J. Opt. B: Quantum Semiclass. Opt. 2, 225 (2000), quant-ph/0002002.

[33] A. O. Pittenger and M. H. Rubin, Note on separability of the werner states in arbitrary dimensions (2000), quant$\mathrm{ph} / 0001110$.

[34] R. Lockhart and M. Steiner, Preserving entanglement under decoherence and sandwiching all separable states (2000), quant-ph/0001110.

[35] W. J. Munro, D. F. V. James, A. G. White, and P. G. Kwiat, Phys. Rev. A 64, 030302 (2001), quantph/0103113.

[36] S. Ishizaka and T. Hiroshima, Phys. Rev. A 62, 022310 (2000), quant-ph/0003023.

[37] F. Verstraete, K. Audenaert, T. D. Bie, and B. D. Moor, Phys. Rev. A 64, 012316 (2001), quant-ph/0011110.

[38] M. L. Mehta, Matrix Theory (Hindustan Publishing, Delhi, 1989).

[39] By "positive measure" we mean that we have divided the Hilbert space into two regions with measures of comparable size in $\mu_{N, m}$, i. e., the ratio of the measures of the two parts is finite (neither infinite nor zero).

[40] For 2 qubits, $\varepsilon_{\text {ent }}$ is at the boundary of the maximal ball (PPT implies separable in this case, see also [30]), which is why the exact results for the bounds for two qubits are the same (r.h.s. of eqs. (8) and (13) are equal). But this isn't so for $d>2$ or $m>2$, hence we get a different value for the bound from this method in general. The states in between are maximally entangled mixed states, see, [35, 36, 37. 